\title{
Interview with Amr H Sawalha: epigenetics and autoimmunity
}

\begin{abstract}
Amr H Sawalha is Professor of Internal Medicine and Marvin and Betty Danto Research Professor of Connective Tissue Research at the University of Michigan, Department of Internal Medicine, Division of Rheumatology. He also holds faculty appointments at the Center for Computational Medicine and Bioinformatics and the Graduate Program in Immunology at the University of Michigan. He was recently appointed as Guest Professor at Central South University in Changsha, China. He received his medical degree from Jordan University of Science and Technology and completed his residency training in internal medicine at the University of Oklahoma Health Sciences Center, and his fellowship in rheumatology at the University of Michigan. His research focus is the genetics and epigenetics of complex autoimmune and inflammatory diseases, including lupus and systemic vasculitis. He has authored over 100 peerreviewed manuscripts, book chapters and review articles, and is on the editorial board of several journals in his field. He has been elected as a member of the American Society for Clinical Investigation, and has received numerous awards, including the Edmund L Dubois, MD, Memorial Lectureship Award from the American College of Rheumatology in recognition for his work in lupus. He is Chair of the Lupus Foundation of America research subcommittee and is a member of the Vasculitis Foundation Medical and Scientific Advisory Board. He also provides clinical care and teaching in the rheumatology outpatient and inpatient services, and he is the director of the NIHfunded rheumatology training grant at the University of Michigan.
\end{abstract}

First draft submitted: 6 January 2017; Accepted for publication: 10 January 2017; Published online: 21 March 2017

Keywords: autoimmunity $\bullet$ biomarker $\bullet$ DNA methylation $\bullet$ epigenetics $\bullet$ epigenomics $\bullet \mathrm{EZH} 2 \bullet \mathrm{FF} 44 \mathrm{~L} \bullet$ lupus $\bullet$ therapeutic

Q Could you please provide a brief summary of your career to date?

I am a rheumatologist and a physician-scientist. I see and treat patients with autoimmune rheumatic diseases and at the same time study the mechanisms of autoimmunity with a focus on genetics and epigenetics. Over the last decade, my work has focused on elucidating the genetic and epigenetic basis of autoimmune diseases, including lupus, systemic vasculitis and Behçet's disease. We utilize genomic, epigenomic and bioinformatics approaches followed by functional studies using in vitro and in vivo systems, to characterize and understand the pathogenic mechanisms of autoimmunity. We have focused on discovering novel genetic and epigenetic susceptibility loci for complex autoimmune diseases, and the functional consequences of these loci to reveal mechanistic insights into disease pathogenesis. We also have performed studies to identify epigenetic changes that can help to understand autoimmune disease subphenotypes, such as disease manifestations [1,2], flares and progression [3,4], and racial differences in disease prevalence and severity [5].
Amr H Sawalha

Division of Rheumatology, Department of Internal Medicine, University of Michigan, Ann Arbor, MI, USA and

Center for Computational Medicine and Bioinformatics, University of Michigan, Ann Arbor, Michigan, USA

Tel.: +1 734763 1858;

Fax: +17347634151

asawalha@umich.edu 
Q How did you become involved in the field of epigenetics?

I started my research career while doing residency training at the University of Oklahoma and the Oklahoma Medical Research Foundation, under the mentorship of John Harley. We were studying the genetics of lupus at that time, initially using linkage studies in families with lupus, and subsequently by candidate gene studies and then genome-wide approaches. I became interested in DNA methylation and the epigenetics of lupus during my fellowship training at the University of Michigan, under the mentorship of Bruce Richardson.

\section{Q Is there hope that epigenetic biomarkers for} lupus may be developed in the near future? Can you describe some of your recent research in this field?

We are gaining significant insight into disease mechanisms by looking at epigenetic changes in lupus. In addition, we are now able to see that different clinical subsets of lupus patients have different DNA methylation signatures. For example, we have recently shown that lupus patients with renal involvement have distinct DNA methylation changes in their $T$ cells that differentiate them from lupus patients without renal involvement [1]. We are also seeing distinct DNA methylation signature in lupus patients with different types of skin involvement [2].

There is definitely hope that specific epigenetic changes can be developed as biomarkers for lupus. A number of epigenetic biomarkers are currently in clinical use in other diseases, such as cancer. In lupus, in a large collaborative effort with Qianjin Lu and his team at Central South University in China, we were able to identify a potential novel diagnostic DNA methylation biomarker for lupus. My research team has previously shown a robust DNA hypomethylation signature in interferon-regulated genes in lupus $\mathrm{T}$ cells and other immune cell types such as neutrophils, including in the gene, IFI44L [6-8]. This demethylation is independent of disease activity [7]. Lu and his team showed that IFI44L demethylation can be detected in whole blood DNA samples from lupus patients, whether the disease is active or not, which is desirable if you are looking for a diagnostic marker. We then tested IFI44L methylation levels as a diagnostic marker in multiple cohorts consisting of large number of lupus patients and healthy controls, and showed that DNA methylation levels of a single CpG site in IFI44L can identify lupus patients with a high sensitivity and specificity [9]. We also showed that it can distinguish between lupus patients and patients with other autoimmune diseases such as Sjogren's syndrome and rheumatoid arthritis [9]. This is perhaps the first potential epigenetic diagnostic marker for lupus, and the results are very promising. Once developed for clinical use, the test can be relatively cheap and not difficult to perform using whole blood DNA which is stable and easy to collect. We are currently working with our colleagues in China for developing this test for clinical use. It is particularly rewarding to me as a physician-scientist to see some of the observations we made in the lab being developed for use in clinical practice.

\section{Q And what about the promise of epigenetics} for the development of novel therapeutics for lupus? Could you again describe some recent research in this area?

I believe it might take some time before we expect to see specific epigenetic modulators as novel therapeutics for lupus. It is important to remember that any potential therapeutic that will result in inhibiting or activating broad epigenetic modulators nonspecifically, might have undesired side effects. I believe a strategy that is more likely to succeed is to find specific diseaserelated targets affected by these epigenetic modulators and then target these specific targets for therapy.

Let me give you an example we are working on, to demonstrate how epigenetics can help us develop novel therapeutic approaches in lupus. We recently characterized DNA methylation changes that happen with lupus flares in naive $\mathrm{CD}^{+} \mathrm{T}$ cells [4]. Lupus is a relapsing remitting disease, and as DNA methylation changes are dynamic, we figured if we understood how DNA methylation patterns change in $\mathrm{CD}^{+} \mathrm{T}$ cells while in the naive $\mathrm{T}$ cell stage, before activation and differentiation, then we can get an idea about early epigenetic events that predispose to disease flares. When lupus was getting more active, we saw an epigenetic landscape shift in these naive $\mathrm{CD}^{+} \mathrm{T}$ cells that favors a specific pattern of $\mathrm{T}$-cell activation and opposes regulatory $\mathrm{T}$-cell differentiation. With bioinformatics analysis followed by microRNA studies in these same cells, we identified increased EZH2, a member of the transcriptional repressive complex PRC2, as a potential driver of this proinflammatory epigenetic shift [4]. We therefore think that inhibiting EZH2 might be worth exploring as a potential therapy for lupus. In fact, inhibitors of EZH2 are being used in clinical trials for cancer. We took our data a step further and identified targets for EZH2 in T cells that might be involved in the pathogenesis of lupus. In work we have not yet published, we found that EZH2 regulates a specific adhesion molecule, which we then found to be overexpressed in lupus T cells. We demonstrated that overexpressing this adhesion molecule in normal $\mathrm{T}$ cells does increase T-cell adhesion to endothelial cells in vitro, and when blocked, can normalize increased T-cell 
adhesion in lupus patients. This is an example of how we can start with a broad epigenetic modulator (EZH2 in this case), and identify more novel specific targets.

Q In your opinion, what have been the most significant breakthroughs technologically \& methodologically that have aided the field of epigenetic research with regard to the study of the immune system/autoimmunity?

The last few years have witnessed an increasing interest and appreciation for the role of epigenetic regulation in the healthy immune system and in autoimmunity. In fact, the complexity of the human genome and chromatin structure, beyond protein coding regions, has become a major focus resulting in the creation of large international collaborative efforts such as ENCODE and the NIH Roadmap Epigenomics Program. Mapping the epigenome across a relatively large number of cell lines and primary cell types and tissues is a significant accomplishment that will help epigenetic and epigenomic studies in both health and disease. With the development of genome-wide DNA methylation array-based technology and high-throughput sequencing, we have now been able to evaluate specific epigenetic marks across the genome in patients with a number of autoimmune diseases, and identify and characterize regions within the genome that are epigenetically altered compared with healthy controls. I think the ability to assess chromatin accessibility, using ATACseq, across the genome using a small number of cells has been a major recent technological breakthrough in epigenetics. We are now also seeing new methods to construct sequencing libraries that will allow for longer sequencing read alignment. This will help to more accurately assess epigenetic changes in regions within the genome that are highly polymorphic and previously less accessible or more difficult to study, such as the HLA region.

Q On the flip side, what obstacles in the current technologies/methodologies present the most significant problems with regard to epigenetic research in lupus? How do you think these issues can be addressed in the future?

I think the main obstacle in lupus epigenetics research is not the technology at this point, but that lupus is a heterogeneous disease. It is very important to realize and incorporate disease heterogeneity into our study designs, whether being epigenetic studies, other mechanistic studies or even clinical trials. It is also important to design and perform longitudinal epigenetic studies in lupus, to study epigenetic changes in individual lupus patients over time. This will help us in many ways, including a more accurate understanding of epigenetic changes in disease progression, epigenetic changes that occur prior to specific lupus manifestations and importantly the time frame between these specific epigenetic changes and disease manifestations. Longitudinal studies will also allow for studying epigenetic changes within the same genetic background and generally more consistent environmental influences in individuals followed over time. Inception cohorts with samples collected from individuals over time before, during and after developing diseases will also be a great way to study epigenetics and their involvement in disease causation and mechanisms, and this is true for any disease, including lupus.

\section{Q Could you give us a glimpse into the research} you are carrying out at present, or have planned for the near future?

We work on elucidating the contribution of both genetic and epigenetic factors to the pathogenesis of a number of immune-mediated diseases, including lupus, Behçet's disease, Takayasu arteritis, scleroderma and others. Our work is very collaborative in nature. Therefore, we set up large collaborative efforts with a number of groups around the world to be most effective in our research efforts. Our focus in the next years will be to translate the knowledge we have obtained from our epigenome-wide studies in autoimmunity, into focused functional studies that will help us understand disease mechanisms and identify novel therapeutic targets. At the same time, we are developing some of our findings into diagnostic tests, and would like to develop epigenetic marks as biomarkers to predict disease relapse and progression in autoimmunity. We also plan to expand on our longitudinal studies and evaluate epigenetic changes over time in specific cell subsets from patients with autoimmune diseases. This has been a work in progress for some time in our lab, and the challenge is to collect sufficient number of patients with samples from multiple time points to be able to analyze these data. We are in the process of evaluating epigenetic modulators (such as EZH2) as a potential therapeutic for lupus starting with an in vivo animal approach.

\section{Q Where do you see the field of epigenetics in 10 years' time?}

In the next 10 years I would expect the field of epigenetics in autoimmunity to move toward more functional epigenetic and epigenomic studies. This will take epigenetic susceptibility loci we have already discovered, and continue to discover, into mechanistic insights for disease causation, pathogenicity and progression. We will see studies that utilize gene editing technologies, such as CRISPR/Cas9, to modify specific epigenetic 
marks in specific loci and study the effect of these epigenetic changes in vitro and in vivo systems. I also think that single cell epigenomic studies will expand and provide new knowledge regarding the epigenetic architecture of specific cell subsets and sub-subsets, and how they contribute to diseases. I envision a more thorough understanding of allele-specific epigenetic changes, and how genetic susceptibility loci in autoimmune diseases interact with and perhaps influence site-specific epigenetic marks to induce pathogenic phenotypes. The interaction between the various epigenetic marks will be more understood in the next 10 years, and how noncoding RNA molecules direct specific epigenetic modulators and transcription machinery molecules will more comprehensively explain regional and locus-specific epigenetic changes. It is an exciting time ahead of us, and the significant advancement in technology and methodological experimental tools coupled with bioinformatics analysis tools, will lead to major discoveries and better understanding of epigenetics and how epigenetic

\section{References}

1 Coit $\mathrm{P}$, Renauer $\mathrm{P}$, Jeffries MA et al. Renal involvement in lupus is characterized by unique DNA methylation changes in naive CD4 ${ }^{+} \mathrm{T}$ cells. J. Autoimmun. 61, 29-35 (2015).

2 Renauer P, Coit P, Jeffries MA et al. DNA methylation patterns in naive $\mathrm{CD} 4^{+} \mathrm{T}$ cells identify epigenetic susceptibility loci for malar rash and discoid rash in systemic lupus erythematosus. Lupus Sci. Med. 2(1), e000101 (2015).

3 Hughes T, Ture-Ozdemir F, Alibaz-Oner F, Coit P, Direskeneli H, Sawalha AH. Epigenome-wide scan identifies a treatment-responsive pattern of altered DNA methylation among cytoskeletal remodeling genes in monocytes and $\mathrm{CD}^{+}{ }^{+} \mathrm{T}$ cells from patients with Behcet's disease. Arthritis Rheumatol. 66(6), 1648-1658 (2014).

4 Coit P, Dozmorov MG, Merrill JT et al. Epigenetic reprogramming in naive $\mathrm{CD}^{+} \mathrm{T}$ cells favoring $\mathrm{T}$ cell activation and non-Th1 effector $\mathrm{T}$ cell immune response as an early event in lupus flares. Arthritis Rheumatol. 68(9), 2200-2209 (2016).

5 Coit P, Ognenovski M, Gensterblum E, MaksimowiczMckinnon K, Wren JD, Sawalha AH. Ethnicity-specific mechanisms and dysregulation contribute to diseases. More importantly, we will better understand how to use this knowledge to better treat our patients and prevent disease complications. It will be a fascinating decade for epigenetics.

\section{Disclaimer}

The opinions expressed in this interview are those of the interviewee and do not necessarily reflect the views of Future Medicine Ltd.

\section{Financial \& competing interests disclosure}

AH Sawalha is listed as inventor on a patent application to use IFI44L methylation as a diagnostic test for lupus. The author has no other relevant affiliations or financial involvement with any organization or entity with a financial interest in or financial conflict with the subject matter or materials discussed in the manuscript apart from those disclosed.

No writing assistance was utilized in the production of this manuscript

epigenetic variation in naive $\mathrm{CD} 4^{+} \mathrm{T}$ cells and the susceptibility to autoimmunity. Epigenetics Chromatin 8, 49 (2015)

6 Jeffries MA, Dozmorov M, Tang Y, Merrill JT, Wren JD, Sawalha AH. Genome-wide DNA methylation patterns in $\mathrm{CD}^{+} \mathrm{T}$ cells from patients with systemic lupus erythematosus. Epigenetics 6(5), 593-601 (2011).

7 Coit $\mathrm{P}$, Jeffries $\mathrm{M}$, Altorok $\mathrm{N}$ et al. Genome-wide DNA methylation study suggests epigenetic accessibility and transcriptional poising of interferon-regulated genes in naive $\mathrm{CD}^{+} \mathrm{T}$ cells from lupus patients. J. Autoimmun. 43, 78-84 (2013)

8 Coit P, Yalavarthi S, Ognenovski M et al. Epigenome profiling reveals significant DNA demethylation of interferon signature genes in lupus neutrophils. J. Autoimmun. 58, 59-66 (2015).

9 Zhao M, Zhou Y, Zhu B et al. IFI44L promoter methylation as a blood biomarker for systemic lupus erythematosus. Ann. Rheum. Dis. 75(11), 1998-2006 (2016). 